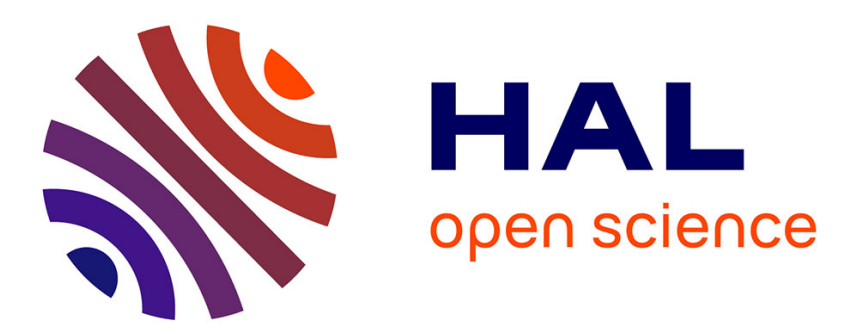

\title{
Influence du climat sur le type de régulation saline du lac Tchad: relations avec les modes de sédimentation lacustre
}

\author{
J.P. Carmouze, G. Pédro
}

\section{- To cite this version:}

J.P. Carmouze, G. Pédro. Influence du climat sur le type de régulation saline du lac Tchad : relations avec les modes de sédimentation lacustre. Sciences Géologiques Bulletin, 1977, 30 (1), pp.33-49. hal-02732231

\section{HAL Id: hal-02732231 \\ https://hal.inrae.fr/hal-02732231}

Submitted on 2 Jun 2020

HAL is a multi-disciplinary open access archive for the deposit and dissemination of scientific research documents, whether they are published or not. The documents may come from teaching and research institutions in France or abroad, or from public or private research centers.
L'archive ouverte pluridisciplinaire HAL, est destinée au dépôt et à la diffusion de documents scientifiques de niveau recherche, publiés ou non, émanant des établissements d'enseignement et de recherche français ou étrangers, des laboratoires publics ou privés. 


\title{
INFLUENCE DU CLIMAT \\ SUR LE TYPE DE RÉGULATION SALINE DU LAC TCHAD RELATIONS AVEC LES MODES DE SÉDIMENTATION LACUSTRE
}

\author{
Jean-Pierre CARMOUZE * et Georges PEDRO **
}

\begin{abstract}
RÉSumé. - La régulation saline du lac Tchad dépend de la régulation hydrique et de l'intervention de processus biogéochimiques par l'intermédiaire desquels certains éléments dissous évoluent vers des formes insolubles. Elle peut être ramenée à la combinaison de deux facteurs antagonistes à composantes multiples. L'un, de nature climato-hydrogéologique, est lié à la régulation hydrique; dans le cas du lac Tchad, il s'agit d'un facteur d'accroissement de la salure des eaux d'origine fluviatile. L'autre, de nature biogéochimique, rend compte de la sédimentation chimique au sein du milieu. C'est un facteur de dessalage.

Ces deux facteurs évoluent de manière opposée en fonction des fluctuations du plan d'eau, ou encore du climat. Leur combinaison est telle que : en période humide, pour des cotes élevées du plan d'eau ( $>$ à 283), le lac est assimilable à un bac de décantation et d'évaporation, dans lequel la salure ne dépasse pas une certaine valeur qui est en grande partie réglée par le premier facteur : la régulation est du type climato-hydrogéologique; en période à caractéristiques climatiques «moyennes», le plan d'eau oscille entre les cotes 279,5 et 283 . Les deux facteurs envisagés s'opposent l'un à l'autre, de sorte que la salure globale reste constante et que le lac fonctionne comme réacteur : la régulation est de type mixte climato-hydrologique et biogéochimique; en période aride, lorsque le plan d'eau se maintient à un faible niveau $(<279,5)$, le lac peut être considéré comme un bac de concentration : la régulation est de type climatique et la sédimentation de nature évaporitique.

Les modes de sédimentation, lorsqu'on considère les trois cas précités, sont dans l’ordre : à dominante «héritée » avec précipitation de calcite, mixte (héritage - dépôts biogènes - néoformations argileuses) et à dominante évaporitique.
\end{abstract}

Sédimentation - Régulation saline - Climat - Lac Tchad

I - INTRODUCTION. GRANDS TRAITS GÉOGRAPHIQUES DU LAC TCHAD

Le bassin du Tchad constitue un vaste système endoréique $\left(2500000 \mathrm{~km}^{2}\right)$, situé au cœur du continent africain entre le $5^{\mathrm{e}}$ et le $25^{\mathrm{e}}$ degré de latitude nord, dans la zone de balancement

* ORSTOM, Section d'Hydrobiologie, 24, rue Bayard, 75008 Paris.

** INRA, Département de Science du Sol, CNRA, 78000 Versailles. 
du front intertropical. Il correspond donc à un milieu continental fermé qui, échappant de ce fait à l'influence marine, est susceptible de subir de profondes variations climatiques, aussi bien à l'échelle de la saison que du millénaire. Ce sont autant de particularités qui en font un milieu privilégié pour l'étude des empreintes climatiques sur les paysages et les milieux.

Au centre du bassin est situé le lac proprement dit. Il correspond à la cuvette terminale du Chari, dont il est en quelque sorte la zone d'épandage puisqu'il ne possède pas d'effluent de surface (fig. 1). Aussi, comme tous les milieux lacustres de steppe, le lac Tchad n'a pas de rives définitives : son niveau et sa surface dépendent étroitement des apports fluviaux, euxmêmes tributaires, dans ces zones arides, de la pluviosité. Il est donc le siège de variations spasmodiques, qui se traduisent par une alternance plus ou moins régulière de pulsations transgressives et régressives.

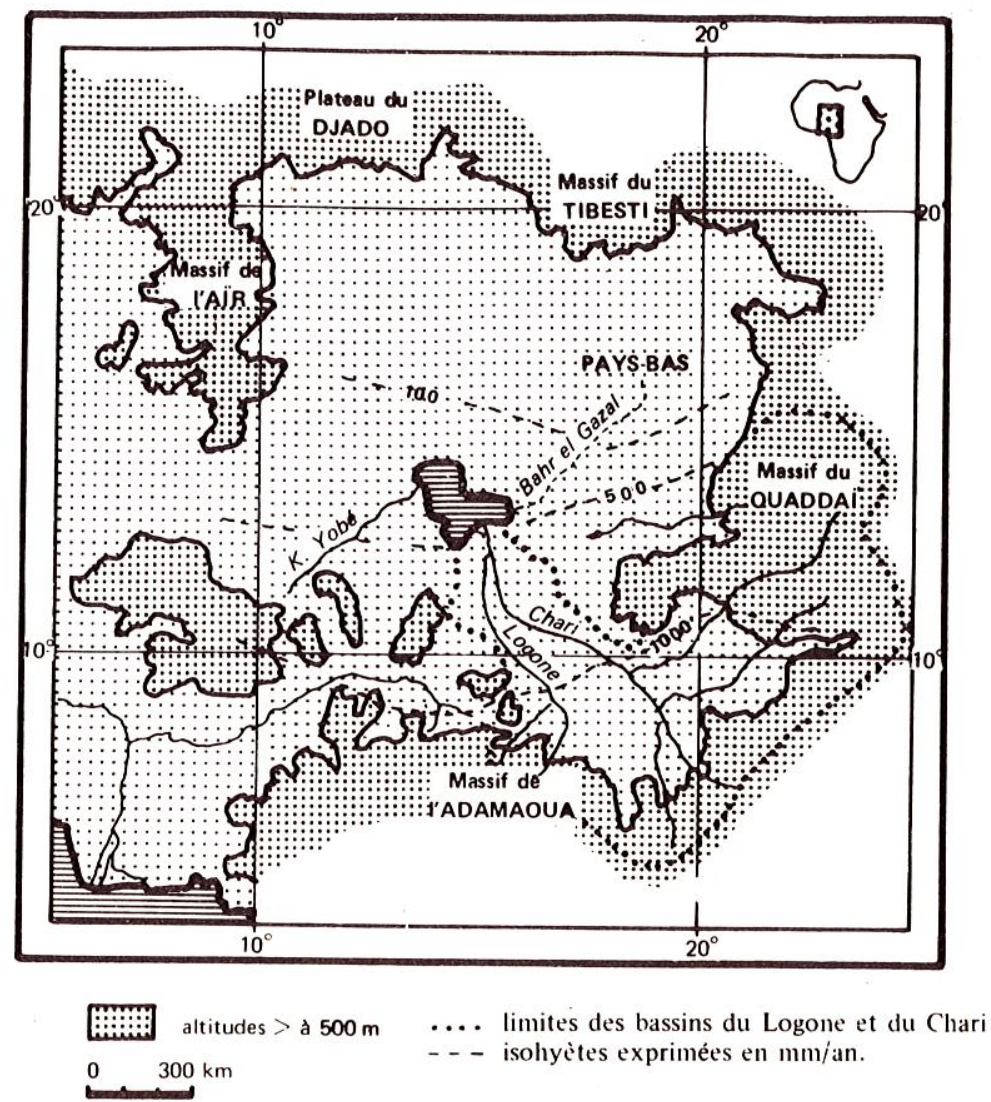

FIG. 1 - Le bassin du Tchad

Depuis l'Holocène supérieur (4 000 ans B.P. - actuel), qui est la période plus directement concernée par cette étude, la cote du plan d'eau a toujours été en dessous de $286 \mathrm{~m}$, de telle sorte que le lac se comporte typiquement comme un lac climatique plat (profondeur de l'ordre de $5 \mathrm{~m}$ ) et qu'il se présente, suivant l'expression de Gautier (1928), comme une "immense daya», dont de vastes zones sont susceptibles d'être exondées ou immergées pour de très faibles fluctuations du niveau. De ce fait, il est possible de rencontrer en fonction du temps un certain nombre de «situations» lacustres; à la suite des travaux de TilHo (1910), on est conduit à en retenir trois principales. 
1. La situation moyenne caractéristique de la période qui va du début du siècle jusqu’à nos jours correspond à une cote voisine de $282 \mathrm{~m}$ : lac moyen. Ce lac s'étend alors sur une surface de $21000 \mathrm{~km}^{2}$ (c'est environ la superficie du lac Balkach), la longueur des grands axes étant comprise entre 100 et $250 \mathrm{~km}$; son volume est de $72.10^{9} \mathrm{~m}^{3}$ et sa profondeur en moyenne de $3,5 \mathrm{~m}$. En réalité cette dernière n'est pas uniforme, car le fond de la cuvette est plus ou moins marqué par un système dunaire. Aussi, suivant la position du sommet des dunes par rapport au plan d'eau, le lac présente-t-il différentes physionomies; c'est pourquoi on a coutume de considérer trois types de paysages, répartis d'ailleurs en deux «cuvettes», sud et nord, qui sont situées de part et d'autre d'une zone de hauts fonds dénommée la « grande barrière » (tableaux I et II). Ce sont :

— un paysage d' îles» lorsque les crêtes des dunes sont exondées : c'est l'archipel (fig. 2 et 3). Le nombre d'îles est de l'ordre de 10-50, la surface moyenne de chacune d'elles étant de $2,4 \mathrm{~km}^{2}$ environ. Au total, cela représente $5600 \mathrm{~km}^{2}$, dont $59 \%$ en eau et $41 \%$ de terre ferme (îles de sable);

— un paysage d' «îles de végétation »: en effet, les crêtes de dunes, lorsqu'elles sont immergées sous 0,50 - 1,50 m d'eau, sont colonisées par des phanérogames semi-aquatiques (Cyperus papyrus et Phragmites principalement). Ce paysage est alors appelé îlots-bancs (fig. 2 et 3 ).

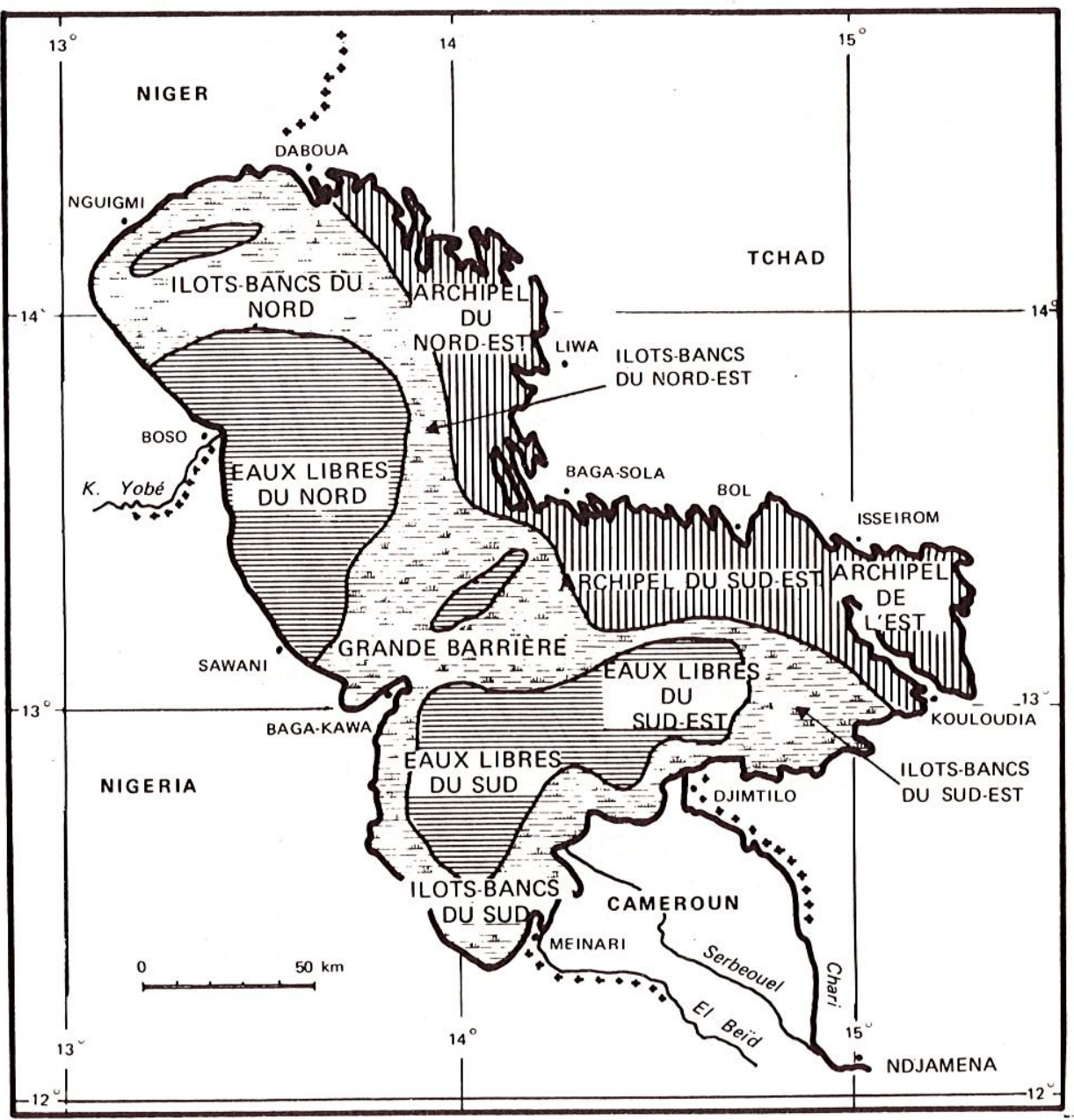

Fig. 2 - Les grandes régions naturelles du lac Tchad. Lac moyen (cote 281,9) 

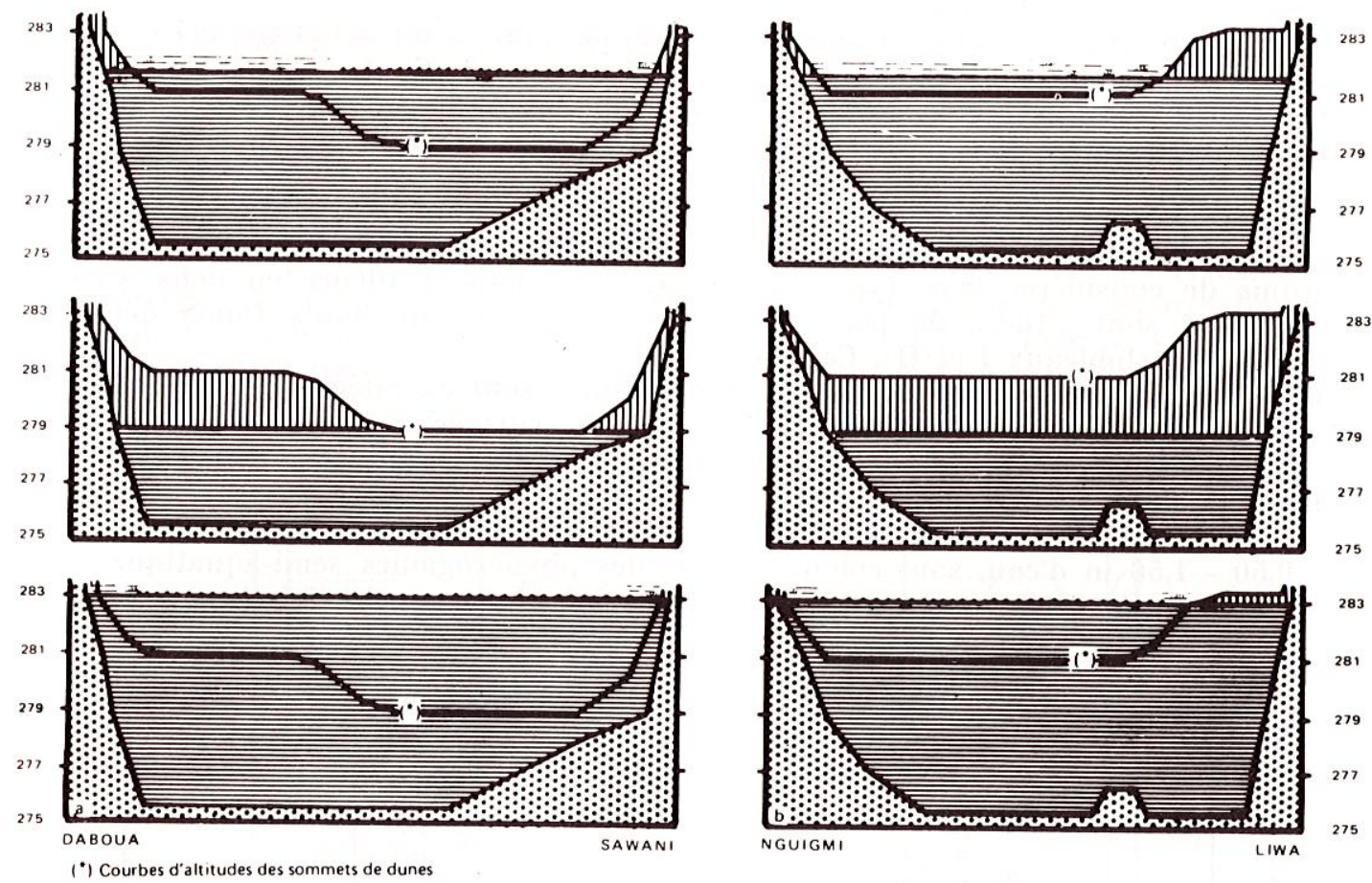

socle tranche d'eau

FIi. 3 a et $\mathbf{b}$ - Courbes topographiques «lissées » suivant deux grands axes de la cuvette nord. De haut en bas : lac moyen (cote 281,8); petit lac (cote 279); grand lac (cote 283)
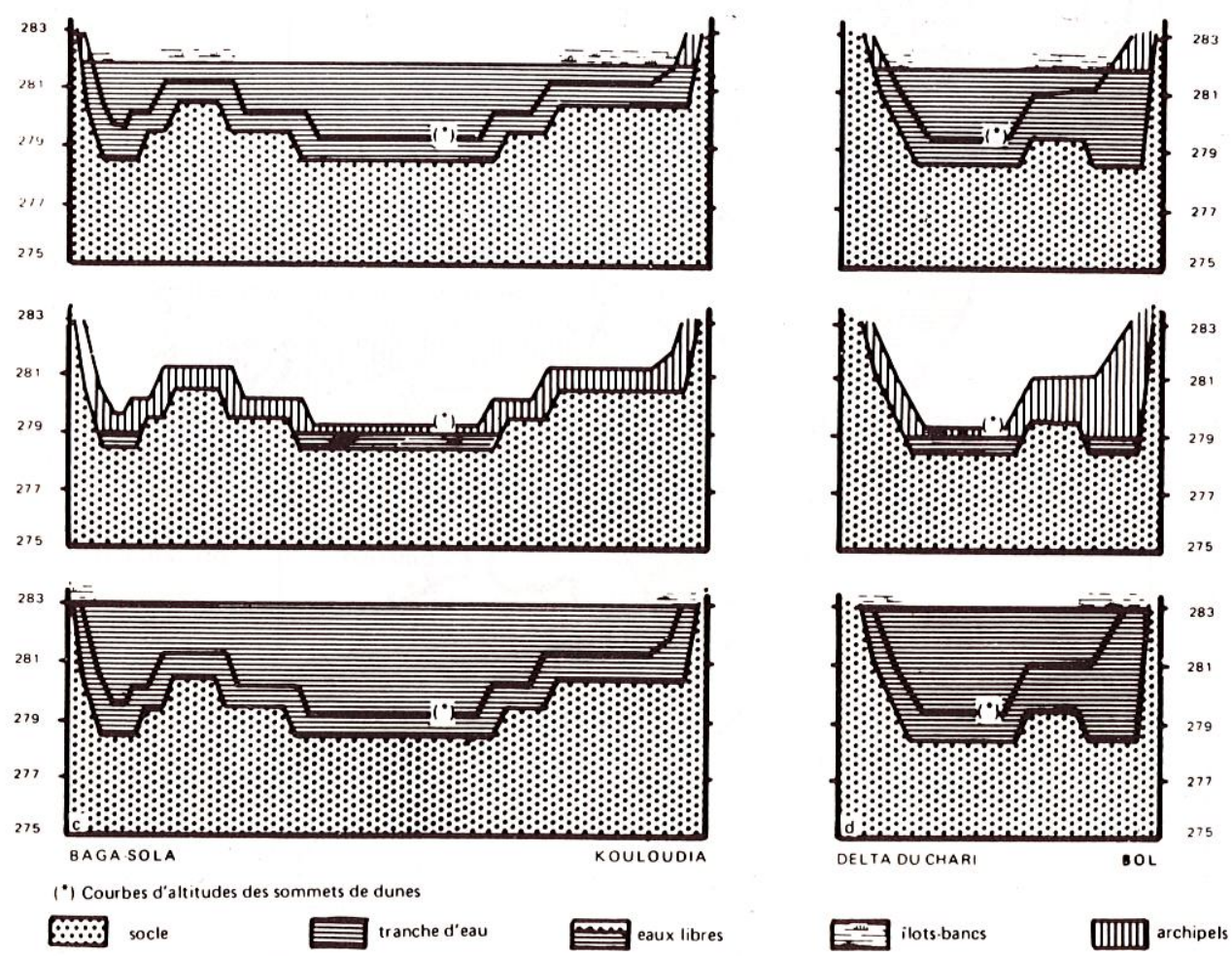

FII. $3 \mathrm{c}$ et d - Courbes topographiques «lissées》 suivant deux grands axes de la cuvette sud. De haut en bas : lac moyen (cote 281,8); petit lac (cote 279); grand lac (cote 283) 
Les îlots-bancs couvrent près de $10000 \mathrm{~km}^{2}$, dont $80 \%$ sont en eau, le reste correspondant à des tapis de macrophytes. Lorsque la profondeur devient inférieure à $0,5 \mathrm{~m}$, il se développe fréquemment des «prairies » à Potamogeton, Vallisneria et Ceratophyllum;

- enfin un paysage d'eaux-libres se manifeste dès que les sommets de dunes sont noyés trop profondément pour qu'il y ait développement de végétation $(>1,50 \mathrm{~m})$; il s'étend en moyenne sur $7700 \mathrm{~km}^{2}$ (fig. 2 et 3 ).

TABLEAU I

Surfaces en eau, volumes et profondeurs moyennes du lac et de ses cuvettes sud et nord

\begin{tabular}{|l|c|c|c|}
\hline & $\begin{array}{c}\text { Surface en eau } \\
\left(\mathrm{km}^{2}\right)\end{array}$ & Volume $\left(\mathrm{m}^{3}\right)$ & $\begin{array}{c}\text { Profondeur } \\
\text { moyenne }(\mathrm{m})\end{array}$ \\
\cline { 2 - 4 } Lac & 21000 & $72 \times 10^{9}$ & 3,40 \\
Cuvette sud & 10800 & $25 \times 10^{9}$ & 2,45 \\
Cuvette nord & 10200 & $47 \times 10^{9}$ & 4,55 \\
\hline
\end{tabular}

Tableau II

Superficies relatives des diverses unités de paysages ( $z$ est la profondeur des hauts-fonds)

\begin{tabular}{|l|c|}
\hline Paysages & Surfaces $\left(\mathrm{km}^{2}\right)$ \\
\hline $\begin{array}{l}\text { Eaux libres } \\
\mathrm{z}>1,5 \mathrm{~m}\end{array}$ & 7700 \\
Ilots-bancs & 10000 \\
$0,5<\mathrm{z}<1,5 \mathrm{~m}$ & (dont $18 \%$ occupés par \\
& les îles de végétation) \\
Archipel & 5600 \\
& (dont $4 \%$ occupés par \\
& les îles de sable) \\
\hline
\end{tabular}

2. Lorsque le niveau d'eau atteint et dépasse la cote $283 \mathrm{~m}$, on a ce que TrLHo a appelé le grand lac. Il s'agit effectivement d'une petite mer intérieure de $25000 \mathrm{~km}^{2}$, dont le volume est de l'ordre de $96-10^{9} \mathrm{~m}^{3}$. La surface des eaux libres augmente considérablement, tandis que la proportion d'îlots-bancs diminue, car la profondeur moyenne du lac dépasse alors 4,50 m (fig. 4). Cette situation a été celle des années 1963-1964 et, d'une façon plus générale, celle des XIV ${ }^{\circ}$ et XVIII ${ }^{\circ}$ siècles (MALEy, 1973).

3. Lorsque, au contraire, le niveau descend en dessous de $279,5 \mathrm{~m}$, le lac se morcelle et la surface tombe en dessous de $10000 \mathrm{~km}^{2}$ (fig. 2). C'est ce qui s'est produit par exemple en 1910 ('TilHO, 1910) et, plus près de nous, en 1973-74 (CARmouze et al., 1973 a et b). La cuvette nord a été alors entièrement asséchée, après être passée par un stade «archipel», puis par un stade présentant un grand nombre de mares bordières isolées (fig. 4). Quant à la cuvette sud, elle s'est rétrécie jusqu'à correspondre à une surface de l'ordre de $2000 \mathrm{~km}^{2}$ (lac Léman : $580 \mathrm{~km}^{2}$ ) et à un volume de $2.10^{9} \mathrm{~m}^{3}$ (fig. 4).

A partir de cet ensemble de données, dont un résumé est présenté dans le tableau III, et disposant en même temps d'un certain nombre d'éléments sur la régulation saline du lac Tchad et, par là même, sur l'intensité et le type des sédimentations chimiques et biochimiques 

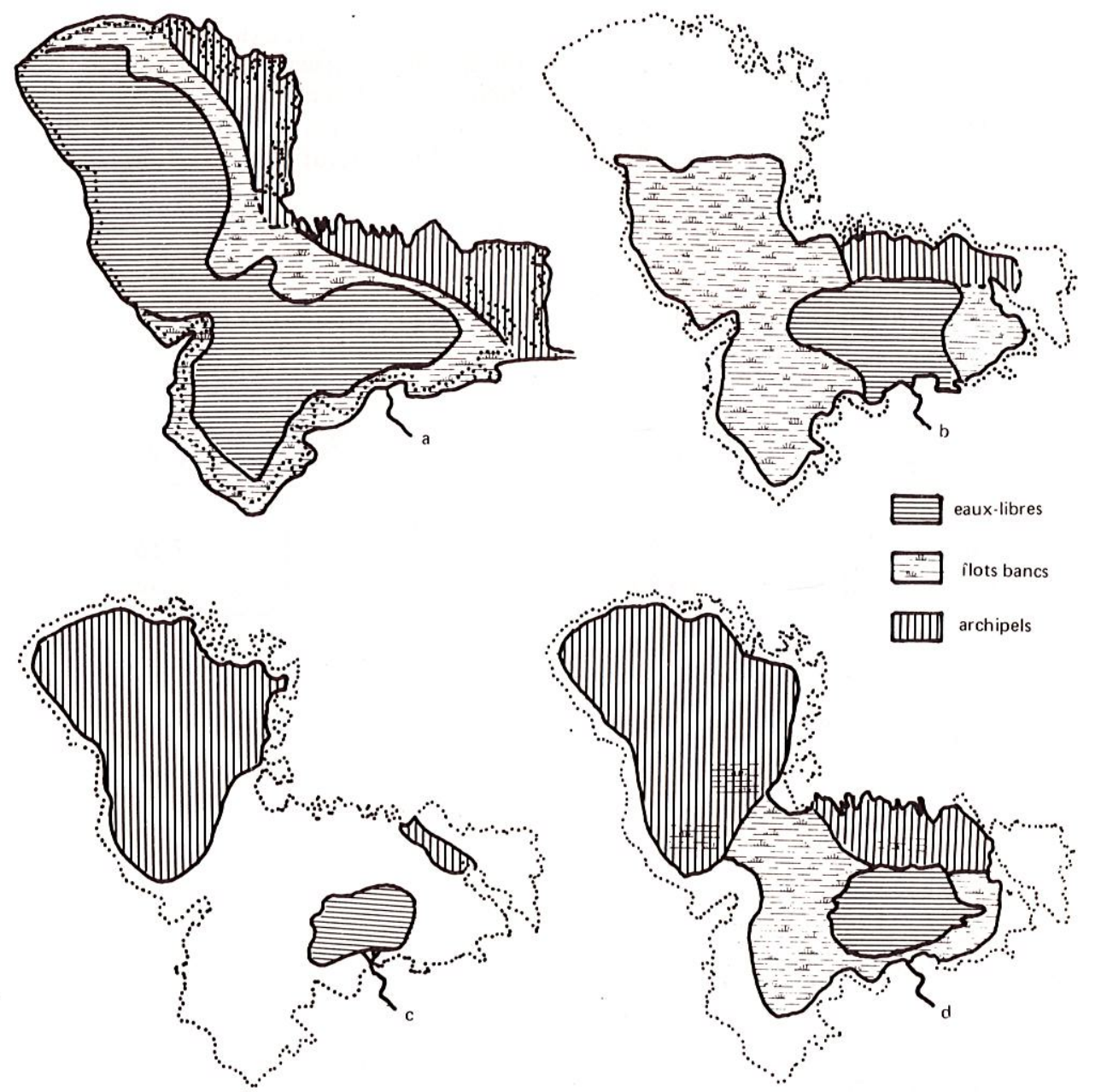

Fig. 4 - Principales physionomies du lac Tchad

a - le «grand 》 lac à la cote 283

b - un «petit» lac : situation en 1908 (TiLнo, 1910)

c - un «petit» lac : situation en juin 1973

d - un «petit» lac : situation en décembre 1973

enregistrées (CARmouze, 1976), nous avons été amenés à aller plus loin dans l'analyse des phénomènes. A ce sujet, le problème plus particulier qui se posait consistait à voir si des relations pouvaient éventuellement exister entre les pulsations transgressives et régressives périodiques du lac actuel et les modes de sédimentation lacustre. C'est ce qui nous a conduits, dans une première phase, à établir les principes de la régulation saline relatifs au lac moyen, avant d'envisager les variations de cette régulation en fonction des fluctuations du plan d'eau, c'est-à-dire finalement des variations climatiques du milieu. 


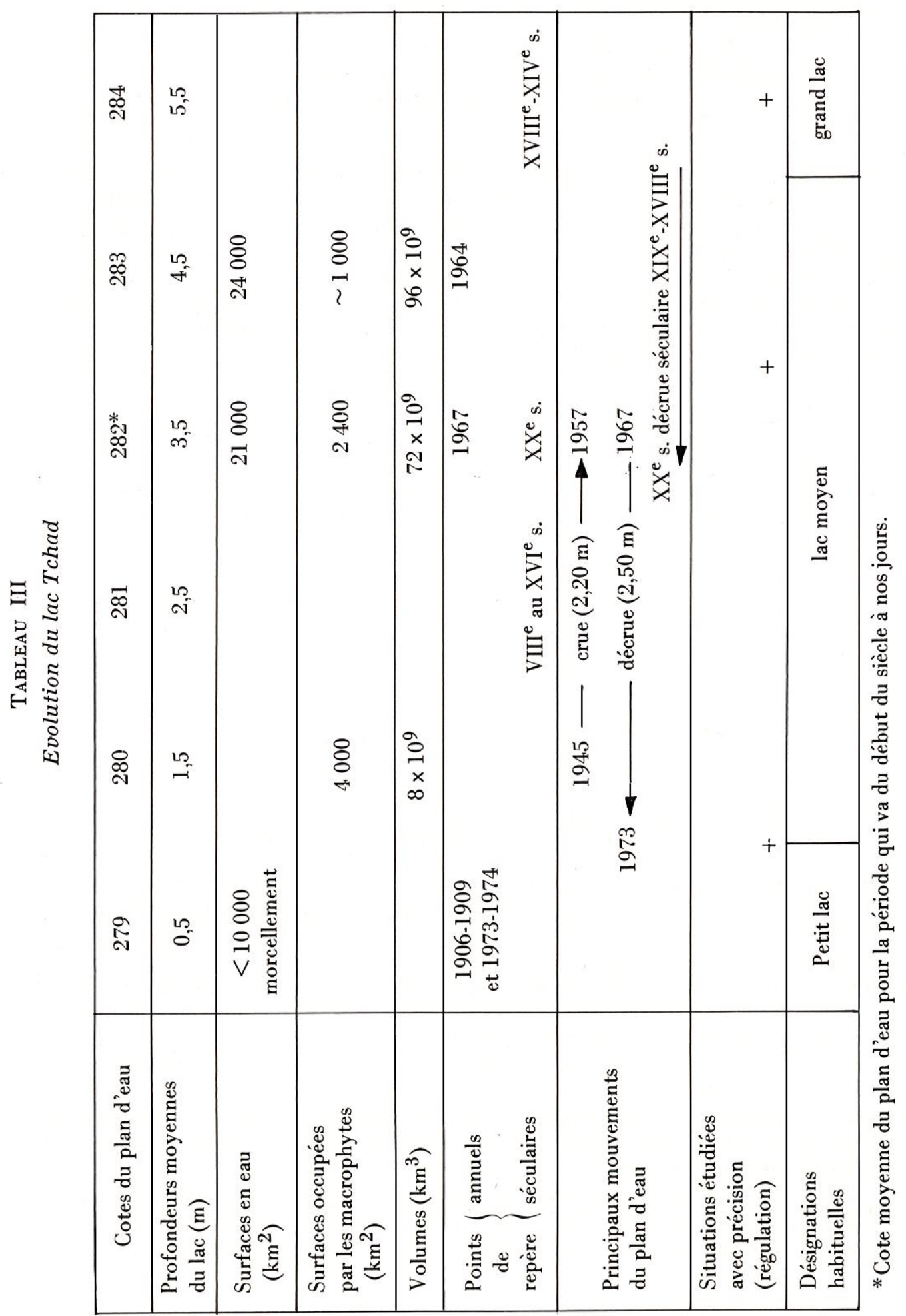




\section{II - LA RÉGULATION SALINE DU LAC TCHAD : MISE EN ÉVIDENCE DES FACTEURS DE RÉGULATION; LEUR IMPORTANCE RELATIVE}

Considéré dans son état moyen, le lac Tchad apparaît comme un système complexe, car il ne constitue ni un simple bassin de concentration, ni un simple bassin de décantation.

$\mathrm{Si}$, en effet, il était un bassin de concentration *, on devrait, du fait de l'intense évaporation qui règne au-dessus de la cuvette $(2,11 \mathrm{~m})$, aboutir à des eaux caractérisées par une assez forte salure. Or il n'en est rien, puisque le Tchad se présente comme un lac d'eau douce : la teneur en éléments dissous est de $363 \mathrm{mg} / \mathrm{l}$ en moyenne. Par rapport aux eaux fluviatiles $(61,5 \mathrm{mg} / \mathrm{l})$, il y a certes concentration, mais celle-ci reste très limitée, le facteur moyen de concentration étant de 5,9 (tableau IV).

Tableau IV

Compositions chimiques moyennes des eaux fluviatiles et lacustres

\begin{tabular}{|l|c|c|c|}
\hline Eléments & $\begin{array}{c}\text { Eaux du Chari } \\
(\mathrm{mg} / \mathrm{l})\end{array}$ & $\begin{array}{c}\text { Eaux du lac } \\
\text { moyen }(1967) \\
(\mathrm{mg} / \mathrm{l})\end{array}$ & $\begin{array}{c}\text { Coefficient } \\
\text { de } \\
\text { concentration }\end{array}$ \\
\hline $\mathrm{Si}(\mathrm{OH})_{4}$ & 22,2 & 46,7 & 2,1 \\
$\mathrm{CO}_{3} \mathrm{H}^{-}$ & 31,5 & 247,7 & 7,8 \\
$\mathrm{Ca}^{2+}$ & 2,06 & 12,50 & 6,0 \\
$\mathrm{Mg}^{2+}$ & 0,94 & 6,20 & 6,6 \\
$\mathrm{Na}^{+}$ & 2,92 & 33,6 & 11,5 \\
$\mathrm{~K}^{+}$ & 1,84 & 16,81 & 9,1 \\
$\mathrm{Total}^{+}$ & 61,50 & 363 & 5,9 \\
\hline
\end{tabular}

Si, d'autre part, il était un simple bassin de décantation, les suspensions solides du Chari et les sédiments superficiels du lac devraient avoir une constitution minéralogique similaire. Or ce n'est pas non plus ce que l'on trouve (tableau V).

En effet, l'examen de ce tableau montre tout d'abord que les teneurs en kaolinite, illite et feldspath, que ce soit dans les charges solides du Chari ou dans les sédiments du lac, sont liées entre elles selon des proportions voisines. Mais relativement à ces minéraux, on note aussi une nette augmentation du taux de smectites. En outre, des dépôts de carbonates résultant à la fois de la précipitation chimique (calcite) et de l'accumulation de coquilles de mollusques (aragonite) sont observés.

Dans ces conditions, on est amené à penser que le lac est le siège de sédimentations chimiques et biochimiques qui concourent à la régulation saline et que, de la sorte, il fonctionne normalement comme un réacteur.

Ceci étant posé, il reste à identifier les processus régulateurs, puis à évaluer leur impact sur l'hydrogéochimie du lac. En réalité, une telle opération ne peut être envisagée qu'à partir d'une description dynamique du milieu, qui permet de tenir compte des échanges de matière.

\footnotetext{
* Dans ce cas, la concentration ne dépend que des pluies, de l'évaporation et des infiltrations.
} 
Tableau V

Composition minéralogique moyenne des transports solides en suspension du Chari et des sédiments du lac

\begin{tabular}{|l|c|c|}
\hline \multicolumn{1}{|c|}{ Minéraux } & $\begin{array}{c}\text { Charge solide } \\
\text { du Chari } \\
\text { (en \% pondéral) }\end{array}$ & $\begin{array}{c}\text { Sédiments du lac } \\
\text { (en \% pondéral) }\end{array}$ \\
\hline Quartz & 9,4 & 1,2 \\
Feldspaths & 5,8 & 4,25 \\
Illite & 11,3 & 7,85 \\
Kaolinite & 38,4 & 26,55 \\
$\mathrm{Smectites}$ & 10,7 & 21 \\
$\mathrm{SiO}_{2}$ amorphe & 2,3 & 4,85 \\
$\mathrm{Al}_{2} \mathrm{O}_{3}$ amorphe & 1,3 & 0,18 \\
$\mathrm{Fe}_{2} \mathrm{O}_{3}$ amorphe & 2,75 & 1,39 \\
$\mathrm{Perte}$ au feu & 18,1 & 22,85 \\
\hline Kaolinite/illite & 6,6 & 6,25 \\
Kaolinite/feldspath & 3,4 & 3,4 \\
\hline
\end{tabular}

Or une description dynamique n'est simple que dans le seul cas où le milieu est considéré en état d'équilibre dynamique, c'est-à-dire fonctionnant en régime permanent. Pour ce qui est du lac Tchad où le régime d'écoulement des eaux fluviatiles varie d'une façon aléatoire, il n'existe pas de parfait ajustement entre les apports et les pertes à l'échelle annuelle; par voie de conséquence, on n'est pratiquement jamais en régime permanent. Toutefois, si l'on considère une période suffisamment longue, l'état hydrochimique du lac oscille autour d'un état moyen, qui peut être assimilé à l'état d'équilibre du milieu par rapport au régime moyen des apports et des pertes relatifs à la même période. C'est la raison pour laquelle nous avons été conduits à retenir deux périodes correspondant respectivement, l'une à un état moyen caractéristique de la cote 282 (c'est la situation qui existait par exemple en 1967), l'autre à un état se rapportant à la cote 283-284.

En se référant à ces divers éléments, il devient alors aisé d'établir que la régulation saline du lac Tchad résulte de l'intervention de trois sortes de facteurs; ceux-ci sont respectivement de nature hydrique, géochimique et biochimique, puisque la salure est contrôlée en définitive par l'existence d'infiltrations, de sédimentation géochimique et de séquestrations biochimiques.

\section{a) Existence d'infiltrations}

Pour les éléments qui ne participent pas à des réactions chimiques, l'eau constitue le seul facteur de régulation; c'est le cas du sodium par exemple*. Or la valeur du rapport des concentrations : (Na) lac/(Na) fleuve n'est que de 12 (cf. tableau IV), ce qui n'est pas excessif si l'on songe à l'importance de l'évaporation dans cette zone; autrement dit, il existe nécessairement des pertes par effluent souterrain, et l'on peut ajouter même que celles-ci sont relativement

* Il a été montré en effet que les sédiments du lac ne s'enrichissaient en sodium, ni par voie géochimique, ni par voie biochimique 
élevées. Les évaluations effectuées conduisent ainsi à montrer que les infiltrations représentent près de $10 \%$ des pertes hydriques (tableau VI).

TABLEAU VI

Eléments du bilan hydrique relatif à la cote moyenne 282

\begin{tabular}{|l|c|l|c|}
\hline \multicolumn{2}{|c|}{ Apports (\%) } & \multicolumn{2}{c|}{ Pertes (\%) } \\
\hline Fleuves & 87 & Evaporation & 92 \\
\hline Lac & 13 & Infiltration & 8 \\
\hline
\end{tabular}

\section{b) Existence de néoformations géochimiques}

D’après les bilans réalisés pour une cote du plan d'eau légèrement supérieure à celle envisagée lors de l'établissement du bilan hydrique (283-284 contre 282), on constate que les néoformations mises en évidence sont essentiellement : des silicates argileux de type nontronite, beidellite ferrifère et montmorillonite, qui captent $34,5 \%$ de la silice, 20,5\% du magnésium et $7 \%$ du calcium apportés annuellement en solution par les eaux fluviatiles; des carbonates de précipitation (calcite), où sont incorporés $26 \%$ du calcium dissous déversé annuellement.

\section{c) Existence de séquestrations biochimiques}

Celles-ci se font par l'intermédiaire des organismes vivants et principalement : des mollusques $^{*}$, qui consomment $18 \%$ du calcium apporté en moyenne chaque année pour l'édification de leurs coquilles; des diatomées ${ }^{*}$, qui utilisent $13,5 \%$ des apports annuels de silice dissoute au lac pour fabriquer leur test; enfin, des macrophytes ${ }^{*}$, qui séquestrent une quantité relativement importante de potassium (9\% des apports).

Au total, grâce à ces deux dernières voies, $50 \%$ de la silice, $66 \%$ du calcium, $29 \%$ du magnésium et $9 \%$ du potassium, apportés par les fleuves à l'état dissous, en moyenne, chaque année, se sédimentent dans le lac. L'importance relative des voies géochimique et biochimique pour les trois principaux éléments $\left(\mathrm{Si}(\mathrm{OH})_{4}, \mathrm{Ca}^{++}\right.$et $\mathrm{Mg}^{++}$) a pu être déterminée et se présente comme il est indiqué dans le tableau VII.

Tableau VII

Importance relative des différents types de sédimentation chimique touchant la silice dissoute, le calcium et le magnésium

\begin{tabular}{|c|c|c|c|}
\hline Eléments & $\begin{array}{l}\text { Sédimentation } \\
\text { biochimique }\end{array}$ & \multicolumn{2}{|c|}{ Sédimentation géochimique } \\
\hline $\mathrm{Si}(\mathrm{OH})_{4}$ & $26,8 \%$ & $73,2 \%$ dont & $\begin{array}{l}90 \% \text { néoformation de } \mathrm{Fe} \text { et } \mathrm{Mg} \\
10 \% \text { néoformation de nontronite }\end{array}$ \\
\hline $\mathrm{Ca}^{2+}$ & $20,5 \%$ & $79,5 \%$ dont & $\begin{array}{l}26 \% \text { néoformation de smectite } \\
74 \% \text { précipitation de calcite }\end{array}$ \\
\hline $\mathrm{Mg}^{2+}$ & $28,5 \%$ & \multicolumn{2}{|c|}{$71,5 \%$ néoformation de smecticte } \\
\hline
\end{tabular}

* Les diverses espèces sont indiquées dans la monographie due à la section d'Hydrobiologie de l'ORSTOM sur les «Grandes zones écologiques du Lac Tchad», Cahiers ORSTOM, série Hydrobiol., VI, 2, 1972, p. 103-169. 


\author{
III - VARIATIONS DE LA RÉGULATION SALINE \\ EN FONCTION DES FLUCTUATIONS DU PLAN D'EAU
}

Du fait de l'existence de diverses fluctuations historiques (tableau III) et, plus spécialement, de celles qui se sont manifestées depuis 1945, nous avons été amenés à apporter une contribution à l'étude de ce problème. Certes, les données dont nous disposons sont plus ou moins précises et pas toujours complètes. Il a été possible néanmoins de dégager quelques idées générales, en s'appuyant sur trois groupes d'informations :

- la comparaison du taux de sédimentation correspondant à l'époque de la formation de la tranche superficielle des sédiments - soit du XVIII ${ }^{\mathrm{e}}$ au XIx ${ }^{\mathrm{e}}$ siècle, c'est-à-dire lorsque le niveau du lac était situé entre $283-284 \mathrm{~m}$ (grand lac : $25000 \mathrm{~km}^{2}$ ) — et de celui caractérisant l'époque actuelle, dont on sait que la cote oscille autour de $282 \mathrm{~m}$ (lac moyen : $21000 \mathrm{~km}^{2}$ );

- la grande crue qui s'est manifestée entre 1945 et 1957 , correspondant à une montée des eaux de 2,20 m; cette crue s'est traduite par une transgression relativement importante, puisque la surface du lac est passée grosso modo de $6000 \mathrm{~km}^{2}$ à $23000 \mathrm{~km}^{2}$ : elle a donc quadruplé;

— enfin, la forte décrue de $2,50 \mathrm{~m}$ qui a affecté le lac entre 1967 et 1974; elle a été à l'origine d'une régression puisque la surface du lac $\left(21000 \mathrm{~km}^{2}\right)$ est tombée à $2500 \mathrm{~km}^{2}$, soit une réduction de superficie de l'ordre de 7.

A partir de cet ensemble de données, et en ce qui concerne la salure globale du lac moyen, on a pu déduire que : entre 1945 et 1957 , bien que le lac soit passé de la cote 280 à la cote 282,5 , la salure a peu diminué : $4,15 \mathrm{~m}$ mé/l à $3,25 \mathrm{mé} / \mathrm{l}^{*}$; entre 1967 et 1972 , le niveau est descendu de la cote 282 à la cote 279,5 , alors que la salure a peu varié : $365 \mathrm{mg} / \mathrm{l}$ en 1967 et $400 \mathrm{mg} / \mathrm{l}$ en 1972 . Il faut ajouter naturellement que, dans le même temps, la composition a évolué vers le pôle bicarbonaté sodique (tableau VIII).

Tableau VIII

Evolution de la composition chimique relative des eaux (en \% molaire)

\begin{tabular}{|c|c|c|c|c|c|c|}
\hline & $\frac{\left[\mathrm{Ca}^{2+}\right]}{\Sigma[\text { cations }]}$ & $\frac{\left[\mathrm{Mg}^{2+}\right]}{\Sigma[\text { cations }]}$ & $\frac{\left[\mathrm{Na}^{+}\right]}{\Sigma \text { c cations] }}$ & $\frac{\left[\mathrm{K}^{+}\right]}{\Sigma[\text { cations }]}$ & $\frac{\left[\mathrm{CO}_{3} \mathrm{H}^{-}\right]^{*}}{\left[\mathrm{CO}_{3} \mathrm{H}^{-}\right]+\left[\mathrm{SiO}_{4} \mathrm{H}_{4}\right]}$ & $\frac{\left[\mathrm{SiO}_{4} \mathrm{H}_{4}\right]}{\left[\mathrm{CO}_{3} \mathrm{H}^{-}+\mathrm{SiO}_{4} \mathrm{H}_{4}\right]}$ \\
\hline 1967 & 20,8 & 16,8 & 47,8 & 14,5 & 84 & 16 \\
1972 & 13,7 & 14,6 & 57,3 & 14,4 & 87,1 & 12,9 \\
\hline
\end{tabular}

Ainsi, entre 279,5 et $283 \mathrm{~m}$, la salure ne varie pratiquement pas. En revanche, elle s'accroìt nettement lorsque la cote descend en dessous de 279,5 et que le lac se fragmente (d'après les évaluations réalisées en se référant aux taux de sédimentation). Le problème qui se pose alors est de tenter d'expliquer cet état de choses en déterminant quantitativement les variations de l'intervention des différents processus régulateurs.

* En fait, il s'agit ici de salures ioniques globales, qui ont été établies à partir de mesures de conductivité. Dans ce cas, les teneurs en silice dissoute ne sont naturellement pas incluses. 
Ainsi, lors d'une décrue, on avait tendance à imaginer une augmentation automatique de la concentration. En réalité, jusqu’à la cote 279,5 , celle-ci n'apparaît pas, du fait de la baisse simultanée des stocks, qui est consécutive à un déficit des apports, à des pertes marginales dues à la formation et à l'isolement de mares bordières et, enfin, à l'augmentation de la sédimentation chimique (néoformations argileuses et calcitiques) et biochimique : la biomasse des macrophytes augmente naturellement avec l'abaissement du plan d'eau (sa surface peut atteindre $4000 \mathrm{~km}^{2}$, cf. tableau III). Il en est de même de celle des mollusques, du moins jusqu'à la cote 280,5; en dessous en effet, elle a tendance à diminuer (LÉvêQue, 1972).

La comparaison des taux de sédimentation entre la cote 283-284 m - époque de constitution des sédiments de surface - et la cote 281-282 m — qui correspond à l'époque actuelle — donne une idée de l'ampleur des variations de la sédimentation chimique (tableau IX). L'examen du tableau IX montre, en outre, si l'on en juge par les taux relatifs du potassium, que la sédimentation biogéochimique est deux fois plus élevée lorsque la cote descend à $281 \mathrm{~m}$.

Tableau IX

Sédimentation chimique moyenne annuelle en \% des apports annuels moyens

\begin{tabular}{|l|c|c|}
\hline \multicolumn{1}{|c|}{ Cote } & $283-284$ & $281-282$ \\
\hline $\mathrm{Si}(\mathrm{OH})_{4}$ & 44,5 & 81,3 \\
$\mathrm{Ca}^{2+}$ & 33,5 & 44,8 \\
$\mathrm{Mg}^{2+}$ & 28,8 & 40,9 \\
$\mathrm{~K}^{+}$ & 8,8 & 19,5 \\
\hline
\end{tabular}

Par ailleurs, il faut tenir compte de ce que, à la suite d'apports déficitaires en eau, le volume du lac diminue moins rapidement que prévu. En effet, de faibles baisses du plan d'eau provoquent de fortes réductions de la surface lacustre et, par là, des pertes par évaporation. Ce phénomène s'ajoute donc à ceux touchant les stocks en éléments dissous pour freiner l'accroissement de la salure des eaux.

- A l'opposé, lors d'une crue, on imaginerait volontiers une baisse de la concentration. En réalité, celle-ci se manifeste peu, au moins jusqu'à la cote 283, comme on l'a constaté entre 1945 et 1957, période au cours de laquelle le lac a pourtant doublé.

Or, ceci résulte de plusieurs éléments qui provoquent tous une augmentation des stocks : par accroissement des apports; par récupération partielle des sels déposés dans les mares bordières, lorsque ces dernières sont à nouveau inondées; par diminution enfin de la sédimentation chimique, que celle-ci soit de nature biochimique (réduction notamment de la biomasse des macrophytes) ou géochimique. A ceci, il faut ajouter que le volume du lac crôit moins rapidement que prévu à la suite des apports excédentaires: la surface du lac augmente dans de grandes proportions; il en est donc de même des pertes par évaporation.

En définitive, la régulation saline du lac Tchad peut être ramenée à la combinaison de deux facteurs de nature antagoniste. Le premier est lié à la régulation hydrique et correspond à un facteur de concentration : il est égal au rapport de la hauteur d'eau éliminée par évaporation $\left(h_{E}\right)$ et par infiltration $\left(h_{I}\right)$, diminuée de celle relative à la pluviosité $\left(h_{M}\right)$, sur la lame d'eau infiltrée $\left(\mathrm{h}_{\mathrm{I}}\right)$, soit :

$$
F=\frac{h_{\mathrm{E}}-\mathrm{h}_{\mathrm{M}}+\mathrm{h}_{\mathrm{I}}}{\mathrm{h}_{\mathrm{I}}}
$$

Il a été désigné sous le nom de facteur de concentration climato-hydrogéologique lorsqu'il y a des infiltrations $\left(h_{E}\right.$ et $h_{M}$ étant d'ordre climatique et $h_{I}$ de nature essentiellement hydrogéolo- 
gique), et de facteur de concentration climatique $\mathrm{si}_{\mathrm{E}}$ et $\mathrm{h}_{\mathrm{M}}$ interviennent seuls. Le second facteur rend compte de la sédimentation chimique ou biogène; d'où le nom de facteur de dessalage biogéochimique.

L'évolution semi-quantitative de l'intervention respective de ces facteurs a pu être évaluée et se présente telle qu'elle est indiquée dans la figure 5. D'après cette figure, les deux facteurs agissent bien en sens opposé. Quant à leur combinaison, elle est alors telle que l'on peut distinguer trois domaines.

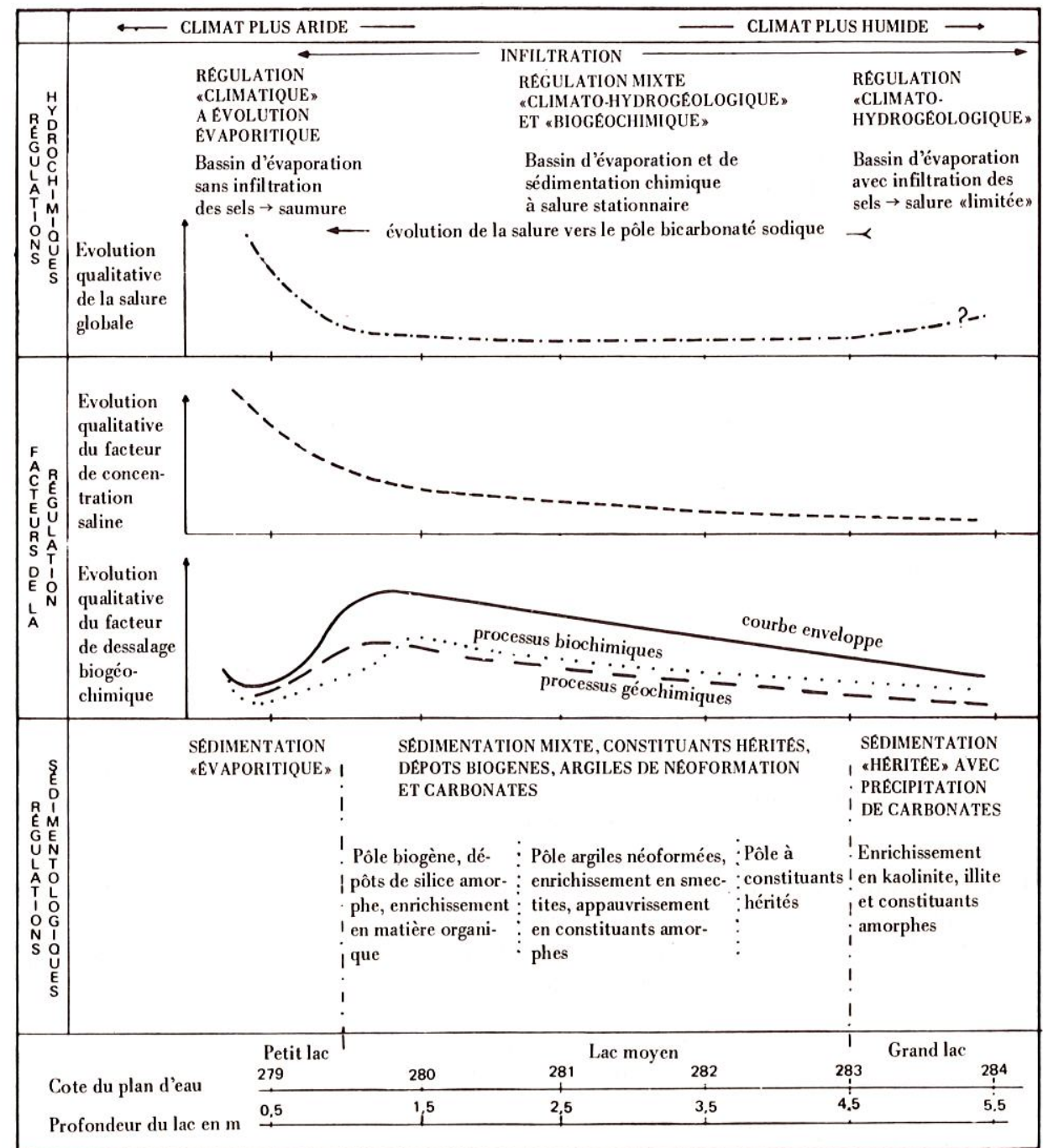

Fig. 5 - Variations des régulations hỵdrogéochimiques et des caractéristiques sédimentologiques en fonction de la cote du plan d'eau

a) Dans le domaine central, situé entre les cotes 279,5 et $283 \mathrm{~m}$ qui correspondent au «lac moyen» au sens large du terme, les facteurs de concentration et de dessalage biogéochimique augmentent de la même façon, au fur et à mesure que le niveau baisse, en sorte que la salure des eaux reste remarquablement constante (la nature de celle-ci évolue néanmoins vers le pôle bicarbonaté sodique). 
b) Lorsque la cote tombe en dessous de 279,5 (ce qui provoque le morcellement du lac), le facteur de concentration croît fortement, du fait que les infiltrations cessent en presque totalité; mais dans le même temps, le dessalage biogéochimique correspondant à l'individualisation de silicates argileux, de silice biogène et de carbonates diminue. La régulation est alors essentiellement assurée par les facteurs climatologiques (pluviosité-évaporation), en sorte que la concentration des eaux augmente fortement.

c) Enfin, quand le niveau dépasse la cote $283 \mathrm{~m}$ et continue à augmenter, les deux facteurs diminuent, mais le facteur de dessalage décroît plus vite que le coefficient de concentration; dans ces conditions, la concentration doit, en tout état de cause, augmenter très légèrement.

\section{IV - CONSÉQUENCES DE LA RÉGULATION LACUSTRE SUR LE PLAN DES PHÉNOMÈNES SÉDIMENTOLOGIQUES}

Si l'on se place maintenant à un point de vue plus strictement sédimentologique, les trois domaines qui viennent d'être décrits peuvent être mis en parallèle avec trois grands types de fonctionnement lacustre.

1. Lorsque le plan d'eau dépasse la valeur 283,5 - $284 \mathrm{~m}$, l'intervention du facteur de concentration climato-hydrogéologique d'une part, et l'influence des processus de néoformation argileuse et de séquestration biogéochimique d'autre part, diminuent; elles deviennent d'ailleurs de moins en moins importantes au fur et à mesure que le plan d'eau s'élève. Dans ces conditions, le lac fonctionne essentiellement comme un bac de décantation, la composition minéralogique des sédiments étant peu modifiée par rapport à celle de la charge solide des fleuves. La sédimentation argileuse est principalement de type détritique (héritage).

2. Entre 283,5 et $279 \mathrm{~m}$, le lac ne se manifeste plus que partiellement comme bac de décantation; il devient plutôt réacteur, avec mise en œuvre de néoformations argileuses, de précipitation de carbonates et de dépôts biogènes. La participation biogéochimique est d'ailleurs d'autant plus importante que le niveau du lac baisse; à $282 \mathrm{~m}$ par exemple, cote pour laquelle nous disposons de données précises, on a pu évaluer que les sédiments chimiques et biogènes représentent pondéralement $30 \%$ et que la sédimentation purement détritique est de $70 \%$. En outre, l'augmentation porte d'abord sur les néoformations géochimiques (argiles-carbonates de précipitation), puis plus particulièrement sur les éléments d'origine organogène (coquilles calcaires et tests siliceux de diatomées). Au total, la sédimentation argileuse est de type mixte (héritage et néoformation).

3. Enfin, en dessous de $\mathbf{2 7 9 , 5} \mathrm{m}$, les apports d'argile détritique d'une part, et les néoformations biogéochimiques d'autre part, diminuent fortement, surtout dans la cuvette nord. Le lac perd alors son rôle de bac de décantation et même, dans une certaine mesure, de réacteur. Les infiltrations ayant cessé, il devient tout simplement une sorte de bac de concentration; lorsque le niveau s'abaisse, la concentration s'accroît donc jusqu'à l'individualisation d'une véritable «saumure », avec mise en place de dépôts évaporitiques de type thermonatrite, trona, gaylussite... (Maglone, 1974). La sédimentation est alors essentiellement chimique et de type évaporitique.

Cet ensemble de données montre avec netteté que la nature des sédiments déposés dans le lac se modifie largement en fonction des fluctuations du plan d'eau. C'est ainsi que, lors d'une pulsation d'ordre régressif, on aurait successivement une sédimentation argileuse de type détritique, puis une sédimentation d'ordre mixte, dans laquelle la part de la néoformation biogéochimique augmenterait au fur et à mesure de l'abaissement du plan d'eau, jusqu'à un certain stade où l'on passerait assez brusquement à une sédimentation de nature exclusivement évaporitique. Au cours d'un épisode transgressif, on obtiendrait naturellement une séquence exactement inverse. 


\section{CONCLUSION}

Dans le cas du lac Tchad, qui est un lac plat situé au centre de la zone aride, il est aisé de montrer que :

1. Tout au moins en dessous de $287 \mathrm{~m}^{*}$, les fluctuations du plan d'eau sont liées essentiellement à des variations climatiques.

2. Les périodes d'humidification du climat correspondent à des phases transgressives et les périodes d'aridification à des épisodes régressifs. En comparant, d'ailleurs, les surfaces maximale et minimale occupées par le lac et les extrêmes pluviométriques enregistrés dans la région, on constate que la surface du lac peut être multipliée par 10 pour une augmentation de la pluviosité annuelle de l'ordre de 40 à $50 \%$ (cf. aussi pour les données historiques TıLHo, 1947).

3. Il suffit, enfin, de très faibles variations dans l'épaisseur de la tranche d'eau, pour passer d'une sédimentation typiquement détritique (bac de décantation) à une sédimentation essentiellement évaporitique (bac de concentration), le stade intermédiaire étant constitué par un système à salure limitée et conduisant à une sédimentation caractérisée par la présence d'argiles de néoformation et de dépôts d'origine biogénique.

Manuscrit déposé le 21 octobre 1976

\section{Abstract}

The salt balance in lake Chad depends upon the hydric regulation and the intervention of biogeochemical processes by which certain dissolved elements evolve towards insoluble forms. The regulation may be understood in terms of the combination of two opposing mult-variate factors :

1) A climato-hydrogeological factor depending on hydric regulation. In the case of lake Chad, this factor is involved in the increase of salinity of fluviatile waters.

2) A factor with a biochemical nature accounting for chemical sedimentation in the milieu. This is a factor of desalting.

These two factors develop (in a contradictory way) as functions of fluctuations in water level or climate. Their combination is such that:

(A) In humid periods for high water levels $(>283$ ), the lake is comparable with a « decantation and evaporation nessel» in which the salt concentration does not exceed a certain value - a value largely determined by the first factor. This type of regulation is of a "climato-hydrogeological " type.

(B) In periods with average climatic characteristics, the water level fluctuates between 279,5 and 283. The two factors considered oppose each other in such a way that the overall salinity remains constant and the lake functions as a "chemical reaction chamber". This regulation is of a mixed "climatohydrological " and "biogeochemical " type.

(C) In dry periods when the water level is low $(<279,5)$, the lake may be considered as a " concentration basin". Under these circumstances, salt regulation is of a climatic type and the sedimentation is evaporitic in nature.

The forms of sedimentation related to the three examples mentioned above are respectively :

(A) A dominant of "heritage" with precipitation of calcite.

(B) A "mixed" form (heritage with biogenetic deposits and argillaceous neoformation).

(C) A dominant " evaporatic" form.

Sedimentation - Salt regulation - Climate - Lake chad

\footnotetext{
* A des cotes supérieures, des phénomènes autres que ceux de nature climatique interviennent (FAURE, 1966; Siefferman , 1970; Servant, 1973).
} 


\section{Zusammenfassung}

Die Regulierung der Salinität des Tchadsees hängt von der Wasserregulierung und dem Zusammenspiel biogeochemischer Prozesse ab, die über Zwischenstadien einige der gelösten Elemente in unlösliche Formen überführen. Sie können auf die Kombination von zwei gegensätzlichen Faktoren mit vielfältigen Verbindungen zurückgeführt werden : der eine von klimato-hydrogeologischer Natur ist an die Wasserregulierung gebùnden; der andere von biogeochemischer Natur weist auf die chemische Sedimentation im Inneren des Milieus hin.

Diese zwei Faktoren wirken entgegengesetzt in Abhängigkeit der Wasserschwankungen und des Klimas : während einer humiden Periode ist der See einem Dekantations- und Evaporationsgefäss ähnlich und die Sedimentation ist überwiegend detritisch mit einer Kalzitfällung; in Zeiten, die durch gemässigtes Klima charakterisiert sind wirkt er als Reaktor und die Sedimentation ist gemischt (detritisch - biogene Ablagerungen - Tonneubildungen); schliesslich, in Trockenzeiten wirkt er als Konzentrationsgefäss und die Sedimentation ist überwiegend evaporitisch.

Sedimentation - Regulierung der Salinität - Tchadsee

\section{Резюме \\ ВАИЯНИЕ КАИМАТА НА ВИА РЕГУАИРОВАНИЯ СОАЕНОСТИ ОЗЕРА ЧАА. ОТНОШЕНИЯ С РАЗАИЧНЫМИ ТИПАМИ ОЗЕРНОГО ОСААКОНАКОПАЕНИЯ}

Регулирование солености озера ЧаА зависит от водного режима и от биогеохимических процессов, посредством которых некоторые растворенные элементы переходят в нерастворимые. Это сводится к сочетанию Авух антагонистических факторов с многочисленными составляющими: один из них, кАимато-гидрологического характера, связан с водным режимом; Аругой биогеохимического характера отражает химическую седиментацию внутри среды.

Эти Ава фактора развиваются противоположным образом в зависимости от колебаний уровня воды или еще от климата. В условиях влажного периода, озеро можно сравнивать с емкостью отстаивания и испарения, а седиментация имеет преимущество, унаследованный характер с выпадением кальцита; в средних климатических условиях, озеро действует как реактор и седиментация будет смешанного типа (унаследованность - биогенные осадки - глинистые новообразования); наконец в аридных периодах оно является емкостью концентрации и, в основном, отлагаются эвапориты.

Седиментация - регудирование солености - климат - озеро ЧаА

\section{BIBLIOGRAPHIE}

Carmouze J.P., Chouret A. et Durand J.R. (1973 a) - Données récentes sur l'évolution du lac Tchad en 1972-1973. Rapport, Centre ORSTOM Fort-Lamy (Ndjamena), multigr., 11 p.

Carmouze J.P., Chouret A. et Franc J. (1973 b) - Assèchement de la cuvette sud du lac Tchad. Rapport, Centre ORSTOM Fort-Lamy (Ndjamena), multigr., 8 p.

Carmouze J.P. (1976) - La régulation hydrogéochimique du lac Tchad. Contribution à l'analyse biogéodynamique d'un système lacustre endoréïque en milieu continental. Thèse Sci. Paris VI, multigr.,
420 p.

FAURE H. (1966) - Evolution des grands lacs sahariens à l'Holocène. Quaternaria, 8, p. 167-175.

Gautier E.F. (1928) - Le Sahara. Payot éd., 232 p. 
LÉvÊQue Ch. (1972) - Mollusques benthiques du lac Tchad; écologie, production et bilans énergétiques. Thèse Sci. Paris VI, 225 p.

Maglione G. (1974) - Géochimie des évaporites et silicates néoformés en milieu continental confiné. Thèse Sci. Paris VI, multigr., 331 p.

Maley J. (1973) - Les variations climatiques dans le bassin du Tchad durant le dernier millénaire : essai d'interprétation climatique de l'Holocène africain. C. R. Acad. Sci., Paris, 273, p. 1673-1676.

Servant M. (1973) - Séquences continentales et variations climatiques. Evolution du bassin du Tchad au Cénozoïque supérieur. Thèse Sci. Paris VI, multigr., 348 p.

Sieffermann G. (1970) - Variations climatiques au Quaternaire dans le sud-ouest de la cuvette tchadienne. $92^{\mathrm{e}}$ congr. Nat. Soc. Sav., Strasbourg, 2, p. 485-494.

Tilho J. (1910) - Documents scientifiques de la mission Tilho (1906-1908). Paris, Impr. Nat., 3 vol.

Tılнo J. (1947) - Le Tchad et la capture du Logone par le Niger. Gauthier-Villars, Paris, 202 p. 